\title{
Cambios morfológicos y sus implicaciones biomecánicas en la rodilla desde el antropoide al hombre
}

\author{
ALBERT ISIDRO \\ Servicio C. Ortopédica y Traumatología \\ Hospital Central Aliança/Barcelona
}

\begin{abstract}
"Don (Donald Johanson), de cuclillas, bajo un sol abrasador, intentaba encajar aquellos dos huesos y de pronto comprendió que aquello no era un mono sino un ser humano"*
\end{abstract}

Maurice Taieb

Esta frase hace referencia al hallazgo de un fémur distal y una tibia proximal, durante la campaña de 1976, en el triángulo de Afar/Ethiopia, correspondiente a un individuo de la especie bautizada posteriormente como Austrolopithecus afarensis, en sedimentos de aproximadamente entre los 3 y los 3.2 millones de años de antigüedad (Plioceno).

Esos dos huesos, que tanto preocuparon a los miembros de la Internacional Afar Research Expedition, eran precisamente los que conforman la articulación de la Rodilla.

El desarrollo de la Bipedestación (BP.) como forma de locomoción predominante hace más de 4 millones de años y, con posterioridad, la práctica de la postura estática bipodal en el Ser Humano; ha condicionado toda una serie de adaptaciones morfomecánicas en la arquitectura global de su diseño anatómico. Las más importantes de ésas adaptaciones, sin lugar a dudas, han sido aquellas que han tenido lugar en su tren inferior.

El pie, probablemente, ha sido la zona anatómica que más cambios ha desarrollado durante el devenir de la BP. Hemos de subrayar que el Hombre es el único animal cuyo peso corporal recae totalmente sobre sus piernas. Los Tetrápodos, por diversos condicionanates biomecánicos centralizan gran parte de su peso en el tren anterior, dejando más libre su tren posterior que se convierte en propulsor. (ej. Camello bactriano 68\%), Los Primates, aún siendo tetrápodos, son los únicos animales cuyo peso recae principalmente en su tren inferior.

Dentro de mundo de los primates reseñaremos la importancia capital en la construcción del diseño corporal, juega el que podríamos denominar «Menú Locomotor». Los Primates, su morfología, su comportamiento y su locomoción están influenciados principalmente por: el Ecosistema y el Peso o Tamaño Corporal

Según varíen estos 2 principales condicionantes podemos citar 7 patrones básicos de locomoción:

\author{
1. Cuadrúpedos Arborícolas \\ 2. Cuadrúpedos Terrestres \\ 3. Saltadores \\ 4. Braquiadores \\ 5. Trepadores \\ 6. Knucle-walkers \\ 7.- Bípedos
}

\author{
(Q. Arb) \\ (Q. Gr) \\ (Leap) \\ (Brach) \\ (CL) \\ $(\mathrm{K}-\mathrm{W})$ \\ (BP)
}

Ningún primate presenta un tipo de locomoción único y exclusivo, a excepción de Homo, pero si muestra un tipo «predominante» de locomoción y otros secundarios u ocasionales.

Asimismo es de gran importancia el tipo postural estático, que adopta en primate cuando descansa o cuando se alimenta; pudiendo ser éstos diferentes que los patrones locomotores, por ejemplo hylobates (gibon) es suspensor ocasional, lo cual, y adelantándonos en el estudio que posteriormente efectuaremos con más profundidad, conlleva aparejado una extensión pasiva de la cadera y de la rodilla; o algunos prosimios como es el caso de los Tarsidiae, muestran lo que podríamos traducir como «aferrado o adherido» a un soporte vertical (vertical clinging) en éste caso particular se presenta una acusada hiperflexión tanto de cadera como de la rodilla, ésta misma flexión de la rodilla la encontramos en las llamadas «eat-feeding postures», postura en este caso pasiva, común en los tetrápodos terrestres tales como los Cercopithecidae.

Entre las variaciones morfológicas encontradas en la rodilla dependientes de su patrón locomotor predominante citaremos a modo de ejemplo las inserciones musculares de los músculos transarticulares que presentan los primates saltadores, mucho más lejanas a la línea articular de la rodilla que las que presentan por ejemplo los primates quadrumanos (ej. saimiri vs. papio).

La rodilla en Homo se caracteriza por su estabilidad en hiperextensión; en la mayoría de primates hay subyacente un cierto grado de inestabilidad de tipo rotacional, circunstancia por la que son mucho más móviles y por tanto adecuadas a los respectivos tipos de locomoción.

Por último creo de gran interés citar las adaptaciones, principalmente de tipo músculo-ligamentosas que presenta la rodilla de los primates que practican habitualmente la Vertical Climbing (ej. Póngidos, Lorísidos etc.). Este patrón locomotor, según hipótesis fiables, funcionalmente es el más parecido, biomecánicamente, 
a la BP. de tipo humano y alejada de aquel tipo de BP. que pueden utilizar, ocasionalmente, algunos primates superiores. La V.CI. conlleva dos tipos de movimientos en las piernas, que son excepcionales en otros tipos de locomoción y estos son: la extensión activa de la cadera y la extensión activa de la rodilla. Estudios basados en métodos electromiográficos y globográficos (Prost, 1980), se observa que la actividad muscular inherente a este movimiento es muy parecida a la que interviene durante la marcha humana.

Son numerosos los trabajos realizados sobre anatomía comparada de la rodilla, tanto en primates extintos como en primates actuales. También son variadas las referencias analíticas de los hallazgos fósiles correspondientes a Homínidos; este trabajo pretende, de forma muy esquemática, dar una visión global desde el punto de vista biomecánico de las diferencias presentes entre la rodilla de los primates, la rodilla de los homínidos y la rodilla del hombre moderno.

Para ello han sido obviados la descripción puntual que han dado a luz los numerosos hallazgos de restos fósiles tanto de primates extintos como de homínidos.

La presencia o no de estructuras anatómicas pertenecientes a la rodilla de aquellos primates cuya crono-filogenia conlleva a la consecución de la BP. y que pertenecen a Aegyptopithecus zeuxi del Oligoceno de AlFayum/Egipto; a Proconsul spp del Mioceno inf. de Africa Oriental y de Kenyapithecus spp del Mioceno medio; presentan indicios que a través del estudio minucioso de toda su anatomía pueden llevarnos a predecir su principal tipo de locomoción y, ergo, extrapolarles a primates actuales.

La estructuración del estudio anatómico comparativo lo dividiremos en cuatro fases:

1. Estudio Comparativo del Extremo Distal del Fémur

2. Estudio Comparativo Extremo Proximal Tibial

3. Estudio Comparativo de la Patela

4. Estudio Comparativo I,igamentos y Meniscos

\section{FÉMUR DISTAL}

La rodilla humana se caracteriza por un alto grado de valguismo en comparación con el resto de los primates.

El componente femoral de éste valgo, queda determinado por el Angulo Femorobicondileo, el cual esta formado por la intersección del eje perpendicular a la línea bicondilar tomada desde el centro del caput femoralis, con el eje diafisario medio del fémur (Walmsley, 1933). Este ángulo en el hombre actual alcanza de medida entre $\operatorname{los} 9^{\circ}$ y los $11^{\circ}$ hacia medial.

Este grado de valguismo representa una modificación importante en la consecución de la BP. al permitir que el pie se sitúe cercano al centro de gravedad corporal durante la fase monopodal de la marcha humana (Kern \& Strauss, 1949; Heiple \& Lovejoy, 1971).

El valguismo es más pronunciado en los niños, situándose entre los $13^{\circ}$ y los $15^{\circ}$ hacia medial entre los 4 y los 5 años (Salenius \& Vankka, 1975). Durante esta edad el eje tibial no es perpendicular a la línea de tierra, por lo tanto el plano articular de la rodilla no es paralelo al suelo. Circunstancia que refuerza la sospecha de que nuestros inmediatos antepasados puedan tener un eje femoro-tibial no paralelo a la línea horizontal (Jenkins, 1972).

El valgo que representa Autstrolopithecus varía según los diferentes individuos estudiados, oscilando entre los $9^{\circ}$ en el individuo grande AL.333.4, hasta los $15^{\circ}$ de individuo pequeño Al. 29.1 (Walker, 1973), ángulos extrapolables a los que presentan los niños entre los 4 y 5 años de edad (ver tabla 1$)$.

TABLA 1

VALGUISMO

ANGULO FEMORO-BICONDILEO

\begin{tabular}{lll}
\hline Homo sap. & $9 / 11^{\circ}$ & (adultos) \\
Homo sap. & $13 / 15^{\circ}$ & (niños 4-5 años) \\
AL.333.4 & $9^{\circ}$ & \\
AL.129.1a & $15^{\circ}$ & \\
TM.1513 & $14^{\circ}$ & \\
STS.34 & $15^{\circ}$ & \\
ATELES/PONGO & $1 / 9^{\circ}$ & (climbers) \\
PAN/GORILLA & $0 /-8^{\circ}$ & (genu varo) \\
\hline
\end{tabular}

Austrolopithecus africanus o gracilis posee un valgo también muy pronunciado cifrándose entre los $14^{\circ}$ de

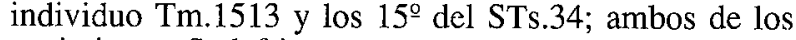
yacimientos Sudafricanos.

Los Primates presentan, en general, una tendencia variante en su rodilla. Esta es más pronunciada en los Póngidos que más relación tienen con el Hombre, oscilando ente $\operatorname{los} 0^{\circ}$ y los $8^{\circ}$ hacia externo. Por el contrario es muy significativo destacar que, los primates cuyo ángulo de varismos es menor y por 10 tanto tienden hacia el valgo de tipo humano son aquellos que practican como patrón locomotor predominante la Vertical Climbing (Heiple \& Lovejoy, 1971; Walker, 1973; Stern \& Susman, 1983).

Por lo que hace referencia al estudio comparativo de la morfología de los Cóndilos Femorales, éste no es de gran utilidad para el conocimiento biomecánico inherente a las características que de ésta zona anatómica se desprende (ver tabla 2).

TABLA 2

CARACTERISTICAS DIFERENCIALES FEMUR MEDIO-DISTAL

\begin{tabular}{llll}
\hline Feature & Homo & Aust & Pan \\
\hline Bicondylar angle & high & very high & low \\
Lat. condyle prof. & elliptic. & elliptical & round \\
Epiphysis shape & square & variable & rectang. \\
Epiphysis symmet. & symmetric & variable & asymmet. \\
Robusticity index & low & high & high \\
Pilasteric index & high & low & low \\
\hline
\end{tabular}


El aspecto morfológico del Surco Patelar o Patella Groove, varía cualitativamente entre los homínidos y el resto de los primates. La característica definitoria de este surco en los homínidos es el resalte del Labio Troclea Externo (LTE). Esta circunstancia morfológica va unida a una mayor longitud, en el plano sagital, de ambos cóndilos femorales.

Visto desde la norma lateral externa el Cóndilo Externo en el Hombre es elíptico, siendo circular en el resto de primates.

Profundizando en aspectos biométricos de ésta zona articular distal del fémur podemos definir dos parámetros útiles desde el punto de vista biomecánico estos son: (Halaczek, 1972)

1. Condylar Length Ratio (CLR)

2. Condylar Tangent Angle (CTA)

El CLR. es el cociente entre la longitud, en el plano sagital, del Condilo Externo y el Condilo Medial.

El CTA. queda definido como el ángulo formado por la línea tangencial que une los dos labios trocleares del surco patelar, con aquella línea que une los puntos más posteriores de los cóndilos femorales. (Ver tabla 3). De su aplicación se deduce que debido a la mayor longitud del cóndilo externo en el hombre, el CLR estará por encima del percentil 1, mientras que su CTA. será en éste caso negativo. (Halaczek, 1972; Tardieu, 1981).

TABLA 3

\section{PARAMETROS BIOMETRICOS} SUPERFICIE CONDILAR FEMORAL

\begin{tabular}{lcc}
\hline & Condil. Length $R /$ & Condil. Tang. Angle \\
\hline Homo sapiens & 1.08 & $-6^{\circ}$ \\
AL.12.1a & 1.03 & $-2^{\circ}$ \\
AL.333.4 & 0.96 & $+3^{\circ}$ \\
\hline
\end{tabular}

En los primates que no pertenecen a la superfamilia Hominidae, tales como los Colobinae y Cercopithecidae (Old Wold monkeys), el labio troclear medial es mayor que el lateral externo (exp del gen. Presbytis). Curiosamente en los New Wold monkeys, el cóndilo mayor es el lateral externo. Citaremos la posible solución alométrica a este supuesto conflicto mencionando que los N.W. monkey o Monos del Nuevo Mundo son casi todos de pequeño tamaño y casi ninguno terrestre (Tardieu, 1981)

La suma de mecanismos morfológicos en una misma rodilla como son la presencia de un Profundo Surco Patelar y un Labio Externo Prominente parece significativo de un mecanismo biomecánico que tiende a prevenir la luxación de la patela hacia externo en una rodilla con un valgo acusado, principalmente durante la hiperextensión (Le Gros Clark, 1947; Heiple \& Lovejoy, 1971; Preuschoft, 1970).

No obstante este mecanismo es puesto en duda (Brattström), aduciendo que durante la hiperextensión de la rodilla con el cuadríceps contraido, únicamente el polo inferior de la patela mantiene alguna relación con la porción más superior o craneal de la superficie articular de la troclea femoral, justo donde no actúa el labio externo prominente. El mismo autor, tras un análisis de la biomecánica femoropatelar, considera que un labio troclear externo prominente previene la subluxación patelar externa solo en ángulos mayores de $20^{\circ}$ de flexión. Así mismo, existe otro mecanismo que limita esa tendencia del cuadríceps (Warnner, 1977).

\section{TIBIA PROXIMAL}

El dato morfológico más significativo al comparar extremidades proximales de la tibia entre los primates y el hombre, es la presencia, en los primeros, de una marcada Retroflexión de la Plataforma Tibial (ver figura 1).

Esta es cuantificable por medio de dos ángulos diferentes

1. Angulo de Retroversión

2. Angulo de Inclinación

El ángulo de retroversión es el formado por el eje diafisario tibial con la tangente a la plataforma tibial superior.

El ángulo de inclinación relaciona la misma tangente de la plataforma tibial con el eje biomecánico de la tibia. (Aiello \& Dean, 1990).

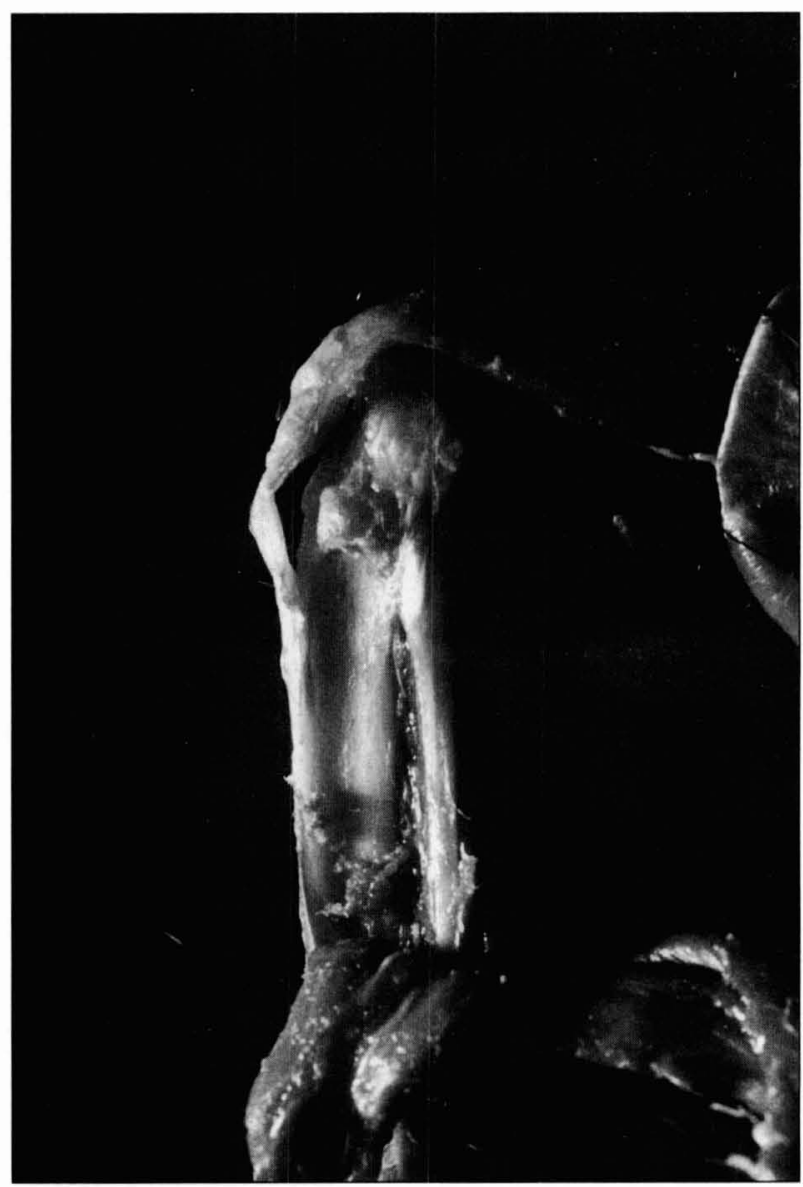

Figura 1. 
En el hombre moderno se puede observar la presencia de una retroflexión tibial superior durante los estadios embrionarios y fetales a causa de la postura genupectoral que éste adopta en el claustro materno.

\section{"El Hombre durante su Vida Embrionaria, trepa a su Arbol Genealógico» T. Huxley (Isidro, 1992)}

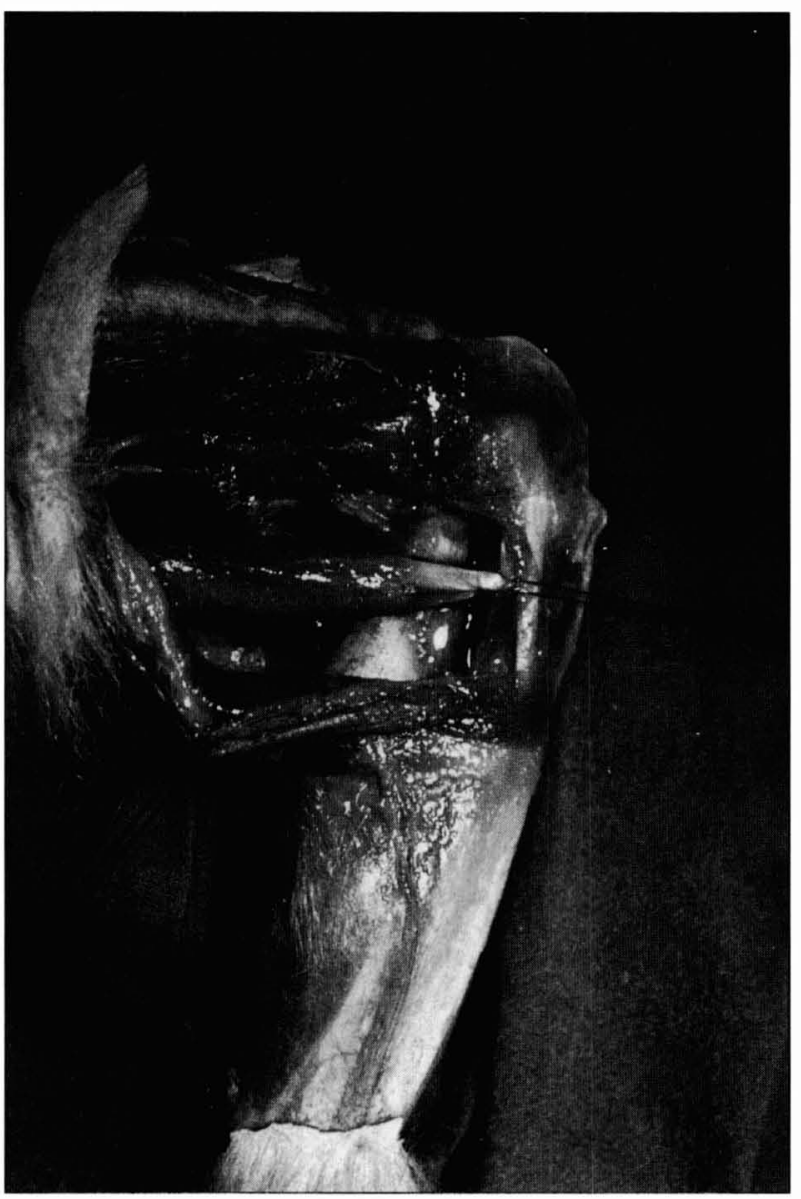

Figura 2.

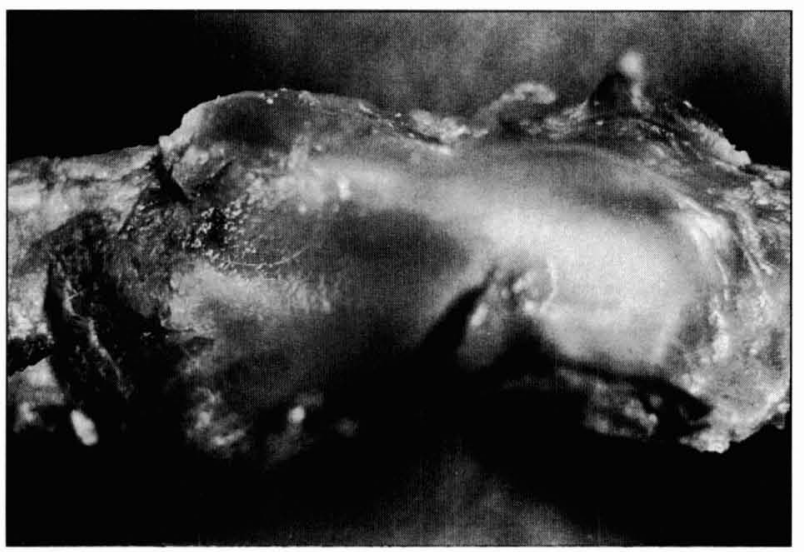

Figura 3.
También existe cierto grado de retroflexión tibial en individuos adultos pertenecientes a étnicas que practican habitualmente la posición de cuclillas.

La repercusión biomecánica de esta retroflexión puede sintetizarse en una reorientación de las fuerzas que actúan sobre las plataformás tibiales, con tal de dirigirlas más perpendicularmente, disminuyendo el stress por cizallamiento en la rodilla (Trinkaus, 1975).

También en las plataformás tibiales hay diferencias significativas entre los primates y el hombre distintas a la de su mayor retroflexión suprametafisaria.

La zona de inserción del M. Semimembranoso, es bastante más distal en lo primates que en el hombre (ver figura 2).

También es diferente la concavidad presente en la zona metafisaria por debajo de la plataforma tibial externa, que es mucho más marcada en los primates que en el hombre.

El M. Tibial anterior presenta una inserción más distal en los primates que en el hombres (Aiello \& Dean, 1990).

También es diferente el Angulo de Torsión Diafisario Tibial, que corresponde al ángulo formado por el eje transversal medio de la plataforma tibial superior con el eje transversal medio de la articulación tibio peronea inferior. Angulo éste que presenta variaciones incluso dentro de homo sapiens según étnias, no obstante podemos decir que este ángulo, en el hombre, se le considera positivo y se situaría en torno a los $+20^{\circ}$. En los Póngidos como Pan y Gorilla, este ángulo de torsión tibial es negativo (alrededor de $-10^{\circ}$ ), mientras que en Pongo pygmaeus (orangután) presenta en rango más extenso oscilando entre los $-25^{\circ}$ y $\operatorname{los}+40^{\circ}$.

\section{PATELLA}

Las diferencias morfológicas que presenta la Patella entre primates y el hombre pueden ser sintetizadas en tres principales:

1. Reducción, tanto Absoluta como Relativa del tamaño. Cuadriceps menos desarrollado en los primates)

2. Diferente inserción del vastus medialis (vs. homo)

3.- Superficie articular plana (ver fig. 3).

\section{LIGAMENTOS Y MENISCOS}

Las diferencias esenciales en lo que respecta a las zonas meniscoligamentarias inciden biomecánicamente al grado de inestabilidad fisiológica de la rodilla en los primates.

Los ligamentos colaterales, tanto el medial como el externo son bastante parecidos a los del hombre, su diferencia radica en su moderadamente inserción más distal, fenómeno que encontramos sobretodo en los primates saltadores (ver fig. 4). 
Los ligamentos que forman parte del pivot de la rodilla, presentan diferencias entre los póngidos y el hombre, centradas principalmente en su dirección y sus zonas de inserción intraarticular, tanto femoral como tibial.

Por lo que respecta al ligamento cruzado anterior (LCA) su dirección, origen e inserciones difieren poco de las que presenta homo. Por el contrario, el ligamento cruzado posterior (LCP), se caracteriza por poseer una dirección mucho más vertical en el sentido cráneo-caudal, diferente de la oblicuidad que presenta homo. Esta disposición paralela al eje sagital, permite a la mayoría de primates el poseer un mayor grado de rotación interna (Corbridge, 1987) (ver fig. 5). Asimismo, es constante la presencia del Ligamento de Wrisberg, que en el primate remeda la dirección del LCP (ver fig. 5), presente solo excepcionalmente en el hombre moderno, se inserta femoralmente en el cóndilo medial justo en la parte superior de la escotadura intercondílea y va a fusionarse distalmente al origen de la zona posterior del mecanismo externo.

Las principales diferencia entre los meniscos se centran:

El menisco externo es completamente circular en el primate, estando unido en su zona posterior al ligamento de Wrisberg; el significado biomecánico de esta zona de inserción meniscal se centra en su actividad durante la

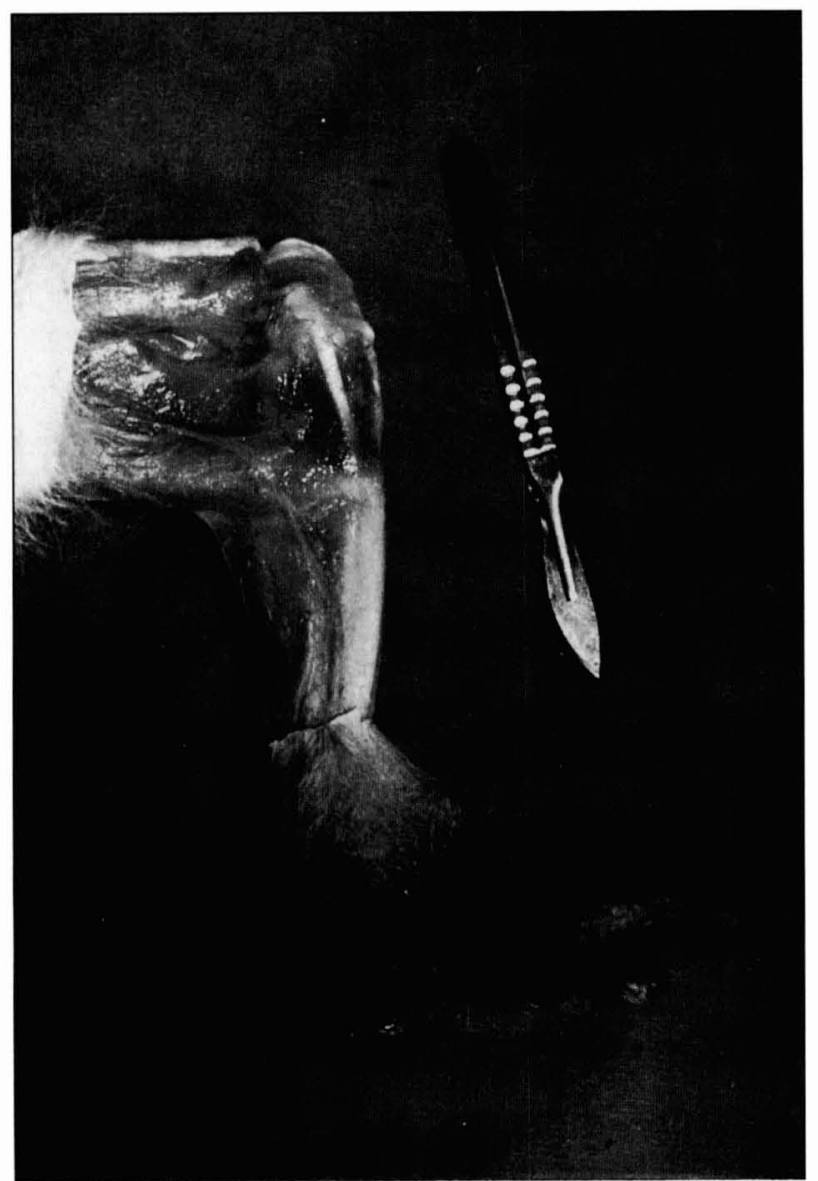

Figura 4. genuflexión al tirar del menisco externo hacia atrás, aumentando durante la hiperextensión el «sliding forward» (Girgis, 1975).

El menisco interno o medial posee un solo punto de inserción, a diferencia de homo que posee dos, que esta situado en la zona tibial posterior. En los primates este menisco medial no esta unido al ligamento colateral medial, estando separado de él por una bolsa serosa.

En los primates también desaparece el ligamento transverso anterior, el cual une, en homo, los cuernos anteriores de ambos meniscos (ver fig. 6) (Aiello \& Dean, 1990).

El mecanismo de cerrojo de la rodilla en hiperextensión que presenta homo para aumentar su grado de estabilidad, esta parcialmente ausente en la mayoría de los primates. Un parámetro antropométrico que, biomecánicamente, pueda representar en términos cuantitativos la congruencia o no de la articulación, así como el grado previsto de rotacionalidad viene dado por la relación entre la distancia Interespinal Tibial (DIST) con la Amplitud de la Escotadura Intercondílea (AEIC), relacionada por la fórmula

\section{DIST x $100 /$ AEIC}

(Tardieu, 1981)

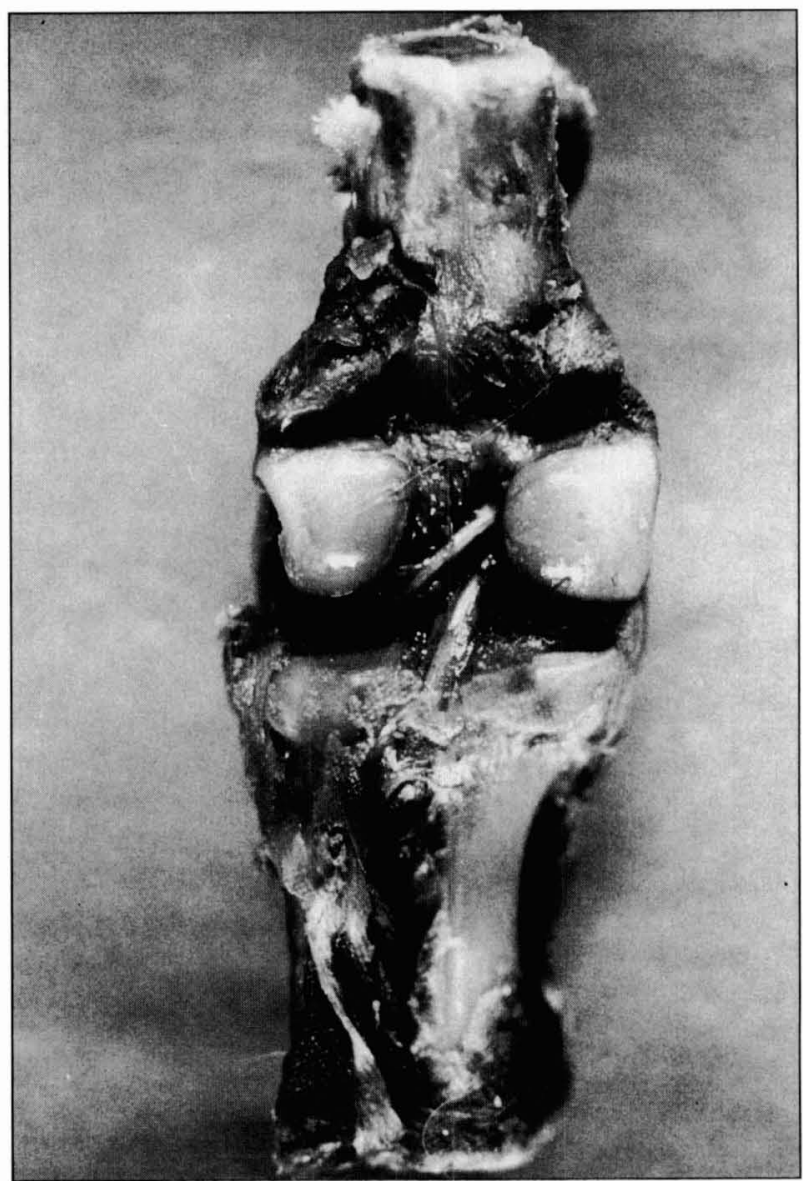

Figura 5. 
El hombre moderno, considerado éste como perteneciente a la subespecie Homo sapiens, posee una escotadura intercondílea estrecha si la comparamos a la amplitud entre las espinas tibiales anterior y posterior, el rango de ésta relación esta situado entre 52 y 75 aprox. Comparado a los demás primates la divergencia es obvia; Gorilla presenta un rango entre 48 y 67 ; Pongo pygmaeus entre 40 y 60 ; Pan troglodytes entre 34 y 55 e Hylobates spp. entre 25 y 40 ; correspondiente a éste último (gibón - diferentes especies) el mayor grado previsto de rotacionalidad.

Por lo que respecta a los primates que practican el quadrupedalismo terrestre los índicces varían entre 30 y 45 en el caso de caarcopithecus spp.

La ventaja de éste medio, es la posibilidad que nos ofrece al aplicarlo a elementos fósiles de la rodilla; si en el caso de Homo sapiens neanderthalensis varía entre los aprox. 68 de individuo de La Ferraise y los 60 en el de La Chapelle aux Saints.

En el caso de los Homínidos más primitivos este índice todavía más; pro ejemplo en Austrolopithecus africanus ER.1481 es de 70, dentro del rango del hombre moderno en su límite superior; mientras que en Austrolopithecus afarensis varia enormemente según el individuo analizado y según su tamaño previsto así, AL.333 presenta un índice de 55; Al.129 de 40 y por último AL.288.1 «Lucy» de 35 , muy por debajo del rango humano moderno, lo cual puede indicar un importante grado de inestabilidad de la rodilla, consecuente con su «menú locomotor» en el cual aún seguía practicando ocasionalmente el Climbing, al trepar a los árboles.

Como hemos mencionado anteriormente hay una clara relación entre el índice estimado o previsto de rotacionalidad y el peso corporal. Si hacemos abstración del Homo sapiens sapiens, observaremos que éste índice es

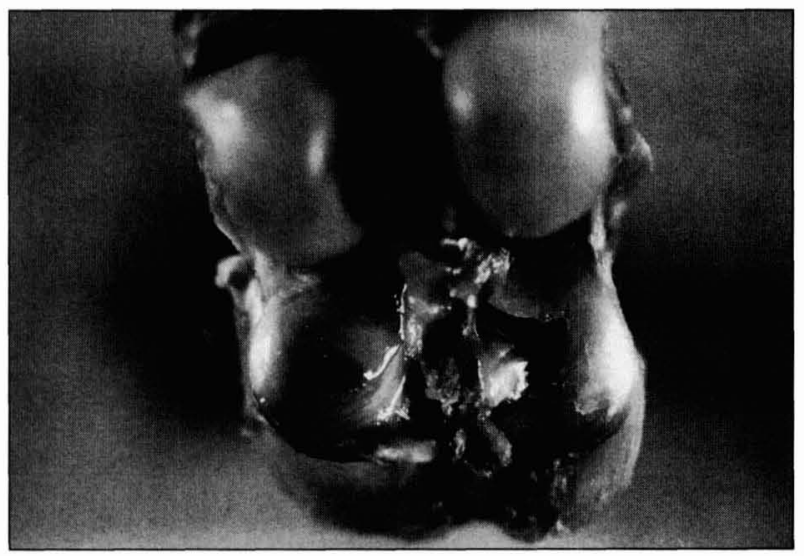

Figura 6. bajo en Hylobates spp (alrededor de 5 a 7 kg.) y máximo en Gorilla gorilla (entre $80 \mathrm{~kg}$ para la hembra y 150 para los machos adultos).

\section{AGRADECIMIENTOS}

Quiero agradecer la colaboración del Dr. Salvador Filella, conservador de Vertebrados del Zoológico de Barcelona por proporcionarme el material necesario para el desarrollo de éste trabajo. Asimismo quiero también agradecer la inestimable colaboración del Dr. Alfredo Zamora con el que pude efectuar la disección del ejemplar que muestran las figuras que aparecen en este trabajo y correpondiente a Cercopithecus neglectus.

\section{BIBLIOGRAFIA}

1. RROST, J.H. (1980) Origin of Bipedalism AM.J.Phys.Anthrop. 52: 175/189

2. WALMSEY, T. (1933) The vertical axes of the femur and their relations. A contibution to the study of the erect posture $J$ Anatom. 67: 284/300

3. KERN, HM \& STRAUS, WL, (1949) The Femur of Plesianthopus transvaaliensis. M.J.Phys.Anthop. 7: 53/77

4. HEIPLE, KG. \& LOVEJOY, CO. (1971) The distal femoral ana tomy of Austrolopithecus Am.J.Phys. Anthop. 35: 75/84

5. SALENIUS, P. \& VANKKA, E. (1975) The development of the tibiofemoral angle in children. J. Bone \& Jo. Surg. 57A: 259/261

6. JENKINS, FA. (1972) Chimpanzee bipedalism Cineradiographyc analysis and implications for the evolution of gait. Science. 178: 877/879

7. WALKER, A. (1973) New Austrolopithecus femora from Eas Rudolph. Kenya J.Human. Evol. 2: 545/555

8. STERN, JT \& SUSMAN, RL. (1983) The locomotor anatomy of Austrolopithecus afarensis. Am.J.Phys.Anthop. 60: 279:317

9. HALACZEK, B. (1972) Die Langknochen der Hinterextremita bei simischen Primaten. Eine vergleichend-mophologische Untersuchung. Dissertation. Anthopological Institute U.Zürich: Juris Druck

10. TARDIEU, C. (1981) Morpho-functional analysis of $y$ the articular surfaces of the knee joint in Primates.

Ina AB Chiarelli and RS. Corruccini (eds): Primate Evolutionary Biology. Berlin: Springer Verlag 68/80

11. CLARK, WE Le Gros. (1949) New paleontological evidence bearing on the evolution of the Hominoidea.

Quart.J.Geol.Soc. 10592): 225/264

12. PREUSCHOFT, H. (1970) Functional anatomy of the lower extremity.

In GH. Bourne (ed): The Chimpanzee. Vol III Basel: Karger pp 221/294

13. BRATTSTROM, H. (1964) Shape of the intercondylar groove normally and in recurrent dislocation of patella.

Act. Orthop. Scand. Supl. 68: 1/148

14. WANNER, JA. (1977) Variations in the anterior patellar groove of the humen femur.

Am.J.Phys. Anthrop. 47: 99/102

15. AIELLO, L. \& DEAN, C. (1990) An introduction to Human Evolutionary Anatomy.

Harcourt Brace Jovanovich, Pub. Acad. Press London

16. ISIDRO, A. (1992) Bipedestación. Cronologia, cauasa y adaptaciones morfomecánicas JIMS (ed) Barcelona.

17. TRINKAUS, E. (1975) Squatting among the Neanderthals: a problem in the behavioral interpretation of skeletal morphology. J.Arch. Sciencies. 2: 327/351

18. CORBRIDGE, R. (1987) The Knee-joint: a functional analys B.Sc. Thesis, University College. London In Stern \& Susman: The locomotor anatomy of Aus. Afarensis. Am.J.Phys.Anthop. 60: pp 298

19. GIRGIS, FG. \& MARSHALL. JL \& AI MONAJEM, ARS (1975) The cruciate ligament of the knee-joint.

Clinic. Orthop. and Rel.Research. 106: $216 / 23$. 